\title{
Turnaround Time for Microbiological Testing of Tuberculosis in Routine Clinical Practice and Time to Patient Initiation on Treatment, Iganga Hospital, Uganda: 2012-2017
}

Angella Musewa ( $\square$ musewaa@musph.ac.ug)

Uganda Public Health Fellowship Program

Lilian Bulage

Uganda Public Health Fellowship Program

Joseph Frank Maganda

Iganga General Hospital

Alex Riolexus Ario

Uganda Public Health Fellowship Program

\section{Research Article}

Keywords: Turnaround time, Microbiological testing, Treatment, MDR Tuberculosis, Uganda

Posted Date: September 9th, 2021

DOl: https://doi.org/10.21203/rs.3.rs-842088/v1

License: (c) (1) This work is licensed under a Creative Commons Attribution 4.0 International License. Read Full License 


\section{Abstract}

Background: Multidrug-resistant tuberculosis (MDR-TB) is caused by Mycobacterium tuberculosis (Mtb) that is resistant to atleast isoniazid and rifampicin. Delayed diagnosis and treatment initiation lead to increased transmission and poor clinical outcomes. Although GeneXpert MTB/RIF detects $M t b$ and rifampicin resistance within 2 hours, the TB control program needs to understand the turnaround time (TAT) from specimen collection to treatment initiation and its impact on treatment outcomes for patients with Rif-resistant TB in Uganda. We quantified the TAT overall and at each step of the process for diagnosis of Rif-resistant TB in routine clinical practice and time to initiation on appropriate treatment over a 5-year period.

Methods: We conducted a retrospective study in Iganga General Hospital, Eastern Uganda. Both Rif-resistant and MDR TB cases are recorded in the same register. We abstracted data from the MDR-TB clinic and laboratory registers (Form 2A and 096B) at Iganga Hospital, 2012-2017, including dates for smear microscopy, Xpert MTB/RIF, initiating MDR-TB patients on treatment after the Xpert MTB/RIF test, and dates for Drug Susceptibility Testing (DST). We performed descriptive and survival analysis to estimate the TAT for microbiological testing and time to initiation on treatment among MDR-TB patients.

Results: Overall, we analyzed sixty-three (63) records, including specimens from Iganga Hospital patients and referrals from other health facilities. Of 63 records, 81\% (51/63) had microscopy results, 97\% (61/63) had Xpert MTB/RIF results, and 98\% (62/63) had DST results. The median TAT for smear microscopy was the same day or within 1 day for 38/51 (75\%) of the patients, ranging from 2-31 days for the rest. TAT for Xpert MTB/RIF results was the same day or within 1 day for 45/61 (75\%), while the rest ranged from 2-183 days. Treatment initiation was within 28 days for 30/61 (50\%). Reporting results for DST, took a median time of 13 days (IQR: 10-40 days) with an overall range of 0-334 days.

Conclusion: Prompt Xpert MTB/RIF testing and result reporting allows timely treatment initiation. We recommend timely release of DST results by the National Tuberculosis Reference Laboratory or decentralization of DST services so as to mitigate delays and improve patient re-evaluation.

\section{Introduction}

Tuberculosis (TB) is the leading infectious cause of mortality worldwide (1). Controlling TB is a key challenge for global public health, and efforts have been hampered by the emergence of drug-resistant strains of Mycobacterium tuberculosis (M. tuberculosis) (2)(3). Multidrug-resistant TB (MDR-TB), defined as disease caused by $M$. tuberculosis that is resistant to at least isoniazid (INH) and rifampicin (Rif), reportedly caused around 240,000 deaths worldwide in 2016, and is associated with poor outcomes despite prolonged treatment (1). Because rifampicin-resistant $M$. tuberculosis is usually simultaneously resistant to $\mathrm{INH}$, rifampicin resistance in tuberculosis (Rif-R) is considered a reasonable proxy for multidrug resistance (MDR) (4).

Recent efforts toward TB control have focused on developing rapid tests for early detection and diagnosis of TB, including drug-resistant TB. The Xpert MTB/RIF ${ }^{T M}$ assay (Xpert, Cepheid, Sunnyvale, CA, USA), an automated, cartridge-based nucleic acid amplification test using reverse transcription-polymerase chain reaction for detection of the M. tuberculosis-specific rpoB gene, represents an important advance in the development of molecular assays to rapidly detecting TB and drug resistance (7). The test identifies $M$. tuberculosis and detects Rif resistance within two hours (8)(9). Xpert was recommended by the World Health Organization (WHO) for the rapid 
detection of TB as well as the detection of drug resistance due to its high accuracy and speed, modest cost, and operational simplicity (7). Current WHO guidelines recommend immediate initiation of MDR-TB treatment (secondline drugs) when resistance is detected by Xpert (1).

Typically, patients are initiated on second-line drugs as they wait for their DST result, which guides the drug regimen. Delays in diagnosis of MDR-TB patients results in amplified drug resistance, precipitating community TB transmission to healthy individuals, and are associated with poor clinical outcomes (5)(6)(1)(10). When MDR-TB patients are initiated on appropriate treatment, sputum culture conversion (from positive to negative) occurs in half of patients within 90 days and in $90 \%$ within 6 months (11).

In 2012, the Ministry of Health Uganda implemented Xpert MTB/Rif in selected facilities, and recommended Xpert MTB/RIF testing for specific populations. These include persons with presumed TB who are HIV-infected and smear-negative, children < 15 years, healthcare workers, and contacts of MDR-TB cases. The test was further recommended for previously-treated smear-positive TB cases to evaluate for rifampin resistance (12). In 2012, WHO recommended targets for turnaround times (TAT) for different microbiological tests. These included 24-hour TAT for smear microscopy, 2-4 weeks for phenotypic DST using solid media, 1-3 weeks for phenotypic DST using liquid culture, and 2 hours for molecular testing (Xpert MTB/RIF) (13). At Iganga Hospital, the MDR-TB treatment unit and Xpert testing were established in 2012. However, the turn-around-time (TAT) from sputum collection to treatment initiation among Rifampicin- resistant patients had not been quantified to inform quality improvement interventions. We quantified TATs overall and for each step in the diagnostic testing of sputum specimens to treatment initiation for presumptive MDR-TB patients.

\section{Methods}

\section{Study Setting}

We conducted the study at Iganga Hospital, a 100-bed general referral hospital serving Iganga and six surrounding districts in Eastern Uganda. The catchment area for the hospital includes >1.5 million persons. Iganga Hospital has been a GeneXpert testing site since 2012 and MDR-TB initiation site since 2013 for TB cases referred from >20 health facilities in Eastern Uganda. Facilities that lack GeneXpert machines refer specimens to Iganga Hospital using the 'hub system,' a national specimen referral network increasing access to critical diagnostic and treatment monitoring services using motorcycles to reduce turn-around times (via 'hub riders'). However, it is frequently applicable when a number of samples (>10) have been collected from patients. After analyzing the specimens at Iganga Hospital, results are communicated immediately to the referring facility through phone call or sent back to the facility using the hub system. The hub riders have specific days for moving to each health facility.

Iganga Hospital has no treatment ward for MDR-TB patients, and instead offers an ambulatory care model for them. Patients who test positive for Rif-resistant M. tuberculosis are initiated on treatment at the MDR-TB treatment center and sent home. Patients are required to visit the hospital for monthly follow-up until they have completed the treatment. Patients who are referred from districts and facilities other than Iganga Hospital are initiated on treatment at facilities close to their residences. A team from the MDR-TB initiation centre in Iganga visits the referring facility and trains the health workers on how the patient is expected to be managed and provides all the necessary medicines. 
Iganga Hospital refers samples for full DST to the National TB Reference Laboratory (NTRL) in Kampala, 155km from Iganga Hospital. Transporting specimens from Iganga to the central laboratory is done through a number of means, including using the national postal courier system and public means with support from implementing partners. Results from DST are then communicated back through an online system to the facility requesting the analysis. This online system is often limited due to issues with internet connection or electricity.

\section{Study Design}

We abstracted data from medical records and laboratory registers at Iganga Hospital as well as its incoming referral network (facilities that refer specimens to Iganga Hospital for TB testing by GeneXpert), for the years 20122017.

\section{Inclusion Criteria}

At Iganga Hospital laboratory, records for specimens submitted for Xpert testing from 2012 to 2017 for presumptive cases of Rif-resistant TB were reviewed, including specimens from Iganga Hospital patients and referrals from other health facilities. We included all presumptive Rif-resistant specimens and those sent to NTRL for phenotypic DST.

We defined a presumptive Rif-resistant case as smear-positive previously-treated TB patients with relapse, return after default (RAD), or failure; new smear-positive pulmonary TB patients from whom the sputum remains smearpositive at month 2 or 3 of treatment; symptomatic close contacts of known MDR-TB patients; and new smearpositive patients.

\section{Study Variables \& Data Collection}

We reviewed laboratory registers records, patient files, and those from MDR-TB treatment unit from 2012-2017 to identify all Rif-resistant and DST results, associated dates, and referring facilities. Variables collected from local facilities from the facility laboratory register included the date smear microscopy was performed in the local laboratory, the date smear microscopy results were obtained from the local laboratory by the TB focal person, the date that the sputum specimen for smear microscopy was received at Iganga Hospital laboratory, and the date the patient started on first-line anti-TB therapy.

Variables collected from Form 2A and 096B (laboratory registers) in Iganga Hospital included date of sputum specimen collection for smear microscopy, date of specimen collection for presumptive MDR-TB at the local facility (referral facilities), date Xpert MTB/RIF test was done at Iganga Hospital, date specimen received in the national reference laboratory for samples from the referral facilities and from Iganga Hospital, date results were reported to referring facilities, and date patient started on appropriate treatment for RIF-resistant TB.

Variables collected from MDR-TB treatment patient files included date when specimen for culture was collected, date when specimen for culture was received in the testing laboratory and processed, date for reporting results to Iganga Hospital. We also estimated the distance from Iganga Hospital to referring facilities using Google maps. We recorded data on a standardized pre-tested form. 


\section{Data Management and Analysis}

Data was entered into a custom Epi Info 7.2 database, cleaned and analyzed using STATA 15.0. Descriptive statistics for TAT were summarized using selected natural percentiles for highly skewed distributions, as well as median, range, and interquartile range. Analysis of each interval was based on specimens with both dates (start and end date) available. TAT was computed for patients to show the number of days for each interval.

To estimate overall TAT for smear microscopy, Xpert MTB/RIF, and time to initiation on treatment, we used KaplanMeier survival analysis. In survival analysis, we also considered specimens for active patients who were lost to follow-up or had missing data allowing for censoring. When patient data (1\%) were lost in the laboratory or not reported to the referral center, the data were removed from analysis.

\section{Results}

\section{Place and Person descriptive characteristics}

We identified and analysed 63 records from Iganga hospital and referral facilities. Of 63 records, 81\% (51/63) records had microscopy results, 97\% (61/63) had Xpert MTB/RIF results, and 98\% (62/63) had DST results (Figure 1). Of 63 records, $76 \%$ (48/63) had results for both Xpert MTB/RIF and smear microscopy, 81\% (51/63) had results for Xpert MTB/RIF and DST, 73\% (46/63) had results for DST and microscopy, and 68\% (43/63) records had results of DST, Xpert MTB/RIF and smear microscopy. Because of missing date values, not all specimens had all of the desired dates recorded (Figure 1).

Most 63\%; 40/63) study specimens belonged to males. The mean age of the patients was 36 years with a range of 6-72. The average distance estimated from the referral facilities to Iganga Hospital was $45 \mathrm{~km}$ with a range of 10$105 \mathrm{~km}$. Most patients were from Iganga, Jinja, Buyende, and Kamuli districts (Table 1).

\section{Table 1}

Demographic characteristics of study participants in Iganga Hospital multidrug-resistant tuberculosis treatment unit, 2012-2017 


\begin{tabular}{lll} 
Variable & Frequency $(\mathbf{n = 6 3})$ & Percentage \\
\hline Sex & & \\
\hline Male & 40 & 63 \\
\hline Female & 23 & 37 \\
\hline District & & 25 \\
\hline Iganga & 16 & 22 \\
\hline Jinja & 14 & 16 \\
\hline Kamuli & 10 & 10 \\
\hline Buyende & 6 & 8 \\
\hline Namutumba & 5 & 5 \\
\hline Mayuge & 3 & 3 \\
\hline Luuka & 2 & 3 \\
\hline Kaliro & 2 & 3 \\
\hline Buikwe & 2 & 3 \\
\hline Bugiri & 2 & 2 \\
\hline Namayingo & 1 & \\
\hline
\end{tabular}

\section{Turnaround time for smear microscopy and first line anti-TB treatment initiation}

Receiving specimens to processing the sample and reporting results for smear microscopy was done within the same day for 38/51 patients (75\%) who had microscopy performed, while 13/51 (25\%) patients had microscopy results the following day. Fifty percent of the patients were initiated on first line TB therapy within one day whereas 13 patients were initiated on first line treatment with a delay of more than 10 days (overall range $0-181$ ) days after receiving results. The overall TAT from specimen collection to initiating patients on first line treatment was a median of only 1 day (overall range 0-181 days, IQR 0-30 days) (Table 2).

\section{Table 2}

Overall time intervals for processing microbiology testing, Iganga Hospital multidrug resistant tuberculosis treatment unit, 2012-2017 
Time Interval

Median

days

(range

25th

Percentile percentile

WHO

$0(0-0)$

0

$0(0-31)$

0

0

$0 \quad$ Immediately

sputum sample in the local laboratory

From receipt of sputum sample to time smear microscopy processed in the local laboratory

From time smear microscopy processed to smear

microscopy result in the local laboratory

$0(0-31) \quad 0 \quad 0 \quad 1-2$ days

From receipt of results to patient initiation on first line treatment

$0(0-181) \quad 0 \quad 10 \quad$ Immediately

From specimen collection to patient initiation on

first line treatment (Overall)

$1(0-181)$

0

30

Immediately

From specimen collection for Xpert to reception in the laboratory

$\begin{array}{llll}0(0-181) & 0 & 2 & \text { Immediately }\end{array}$

From sample collection in the laboratory to analysis

$\begin{array}{llll}0(0-61) & 0 & 1 & \text { Immediately }\end{array}$

From time specimen received to time GeneXpert result are released

$0(0-183) \quad 0$

32

2 Hours

From time result is reported to patient initiation on second line treatment

$28(0$

612)

9

72

Within 7 days

From specimen collection to patient initiation on second line treatment (Overall)

49(0-612)

15

100

Within 7 days

From sample collection for DST to reception of sample

$3(0-367)$

4

Not specified, Varies from

Region to Region

From time the culture is received in the laboratory to the time results are available

$85(2-$

413)

71

$13(0-$

334)

From time the culture results are available to when they are reported to facility

Within 8 weeks

\section{Turnaround time for Xpert MTB/RIF result reporting and initiating patients on second line treatment}

Receiving specimens to processing the sample and reporting results for Xpert MTB/RIF was done within the same day for 46/61 patients (75\%) who had Xpert MTB/RIF performed. Fifteen (25\%) patients received their results with delays of more than 32 days.

Despite the speed of testing for most patients, only 15 (25\%) patients were started on treatment within 9 days of the Xpert results, 31 (50\%) within 28 days, and 15 (25\%) more than 1 month after the Xpert result was reported. The TAT from specimen collection for Xpert MTB/RIF to initiating patients on second line treatment was median of 49 days (range 0-612) days (Table 2). 


\section{Turnaround time for drug susceptibility testing, processing, and reporting by the National Tuberculosis Reference Laboratory}

Turnaround time from sample collection to receiving the specimen for DST was 1 day for 15 (25\%) patients, 3 days for $31(50 \%)$ patients, and 4 days for 15 (25\%) patients. From receiving specimens to processing the sample and reporting results for DST, TAT was 71 days for 15 (25\%) patients, 85 days for 31 (50\%) patients, with delays of more than 116 days for 15 (25\%) patients (Table 2).

Trend for Xpert MTB/RIF turnaround time and treatment initiation

There was no clear trend for TAT of Xpert MTB/RIF, initiation on second line treatment and DST from 2012-2017. The median TAT of Xpert MTB/RIF was 0 days in 2012, increased to 26 days in 2013, and gradually decreased from 2014-2017. The TAT for treatment initiation was low in 2012 and 2014, increased in 2014 and gradually decreased to 7 days in 2017 (Figure 2). The turnaround for DST (gray line) highlights huge delays which impacts decision making for patients with resistance. It is recommended that timely reporting of drug susceptibility testing is paramount to avoid subjecting patients with resistance to inadequate standard first-line treatment for months while awaiting DST results. Usually, patients are initiated on second line as wait for DST result to tell the resistant partner which guides the formulation of proper/suitable regimen for the patient. Exposures to inadequate therapy results in amplified drug resistance and ongoing transmission to healthy individuals. (Figure 2).

\section{Survival analysis curves for turnaround time of smear microscopy: From sample collection to reporting results}

Among fifty (51) records analyzed, median TAT from specimen collection to reporting smear microscopy results was 0 days; 12 of 51 were censored (Figure 3).

\section{Survival analysis curves for turnaround time of Xpert MTB/RIF from sample collection to reporting results to referral facility, 2012-2017}

In total sixty-one (61) records were analyzed, 57 records had all the observations and 4 patients were censored (died before receiving results). The median TAT, from specimen collection to reporting results was 0 days (range 0 648 days) (Figure 4).

\section{Survival analysis curves for turnaround time from sample collection to initiation of patients on second line treatment}

Among 59 records analyzed, median TAT was 50 (0-650) days. Using Kaplan Meier method, we estimated TAT by days in the laboratory, 55 patients had all observations and only four patients died before being initiated on treatment (Figure 5). 
In an analysis of records from 63 MDR-TB patients in Iganga Hospital over a 5-year period, three-quarters of patients received their smear microscopy results in one day or less. Similarly, TAT time for Xpert MTB/RIF from sample processing in the laboratory to reporting results was also within the same day for three-quarters of patients. However, there were delays of more than four weeks for $75 \%$ of patients initiated on second-line treatment. In addition, processing of samples for DST also demonstrated nearly four times the targeted timing ( 116 days vs the recommended 30 days or less). Half of specimens take at least 3 days from specimen collection to receipt at the central laboratory for analysis.

The delays identified in reporting Xpert MTB/RIF results at Iganga Hospital for $25 \%$ of the patients exceeded those recommended by WHO (2 hours). The functionality of the Xpert machines in Uganda has been hampered by unreliable patient referral system, incomplete linkages to TB treatment, and an unreliable infrastructure (such as electrical system malfunctions and poor transportation networks), making implementation of Xpert MTB/RIF more challenging (15).

The WHO recommends immediate initiation of MDR-TB patients on treatment as soon as rifampicin resistance is identified. However, this study reported significant delays of more than 70 days for initiating patients on secondline treatment. This can impact community transmission of drug-resistant TB to healthy individuals.

The TAT for DST from specimen requisition to processing and reporting results was 71 days for 15 patients, 85 days for 31 patients with delays of more than 116 days for 15 patients. This highlights significant delays compared to the standard guidelines by WHO of 2-4 weeks for phenotypic DST using solid media and 1-3 weeks for phenotypic DST using liquid culture (13). A study conducted in Peru reported TAT for DST of approximately 80 days from processing to reporting results, whereas that conducted in Korea reported shorter the TAT for DST at 78 days compared to that reported in our study (14) (17). The trend of TAT for Xpert MTB/RIF testing decreased from 2012 to 2017, although it has not yet met the required TAT as recommended by WHO of 2 hours (13). However, the improvement or reduction in TAT could be attributed to the continuous training of laboratory personnel who perform the Xpert MTB/RIF test, and/or to improvement in infrastructure including reduction in power shortages. The TAT for treatment initiation has also decreased but has not yet met the targets of immediate initiation as recommended by WHO. Observed improvement could be attributed to the improved drug stocks and advances in the Hospital management. During the past year, the body responsible for supplying drugs, the Uganda National Medical Stores had limited drug stocks.

In 2012, the TAT was rapid due to the presence of a TB implementing program that provided transport services for samples to be taken for analysis. In 2013, the program ended, resulting in an increase in TAT. Using this kind of transport requires the MDR-TB coordinator to get the samples to the laboratory on time since the hub system usually has a timetable for which samples are collected and taken to the laboratory. Delays or disruptions in transportation services may impact referral of these patients to diagnostic and or treatment units, delaying initiation of treatment and increasing the risk of TB transmission.

Identifying and addressing delays in transporting samples should be done routinely at health facility level or National Level quality control department to improve the implementation of interventions in resource constrained settings. Strategies that can be employed include decentralizing DST services to avoid long waiting hour from sample collection, transportation and processing at the central laboratory. In addition, there should be proper communication channels between the TB focal persons in the referral facilities and the laboratory where specimen processing is done. Similarly, there is need to improve the available infrastructure such as laboratory 
refurbishment, providing refresher training to laboratory personnel who are responsible for running samples. There should be policy restrictions on timely diagnosis such as reporting microscopy results within $24 \mathrm{~h}$, culture within 21 days, and DST within a month based on the WHO recommendations stated above (13). In addition, internet-based electronic laboratory result system should be adapted to improve TAT, and inform treatment initiation for patients who test positive.

\section{Limitations}

During this study, we were unable to point out the causes of delays at the different intervals. This is due to the nature of the registers used which have no provision for such data to be collected. Since the study was performed at a single site, Iganga Hospital, it is unknown whether the results generalize to Uganda as a whole.

\section{Conclusion}

The TAT for smear microscopy was same day for majority of patients within the study period. However, there were delays in reporting Xpert MTB/RIF results, initiating patients on second line treatment, and reporting DST results to health facilities. We recommend that detailed investigation should be done to find out the stages at which delays occur and the root causes of the delays. Also, similar studies should be conducted in other settings or districts to establish their TAT. In addition, there should be prompt Xpert MTB/RIF testing and result reporting to allow timely treatment initiation. We also recommend timely release of DST results by the National Tuberculosis Reference Laboratory or decentralization of DST services so as to mitigate delays and improve patient re-evaluation. A further analysis of the root causes for the delays at the different stages of testing and result reporting will help focus interventions to reduce of the delays.

\section{Abbreviations}

TB: Tuberculosis; MTB: Mycobacterium Tuberculosis; TAT: Turnaround Time; INH: Isoniazid; DST: Drug Susceptibility Testing; MDR-TB: Multi Drug Resistant Tuberculosis; RMP: Rifampicin; RIF: Rifampicin; NTRL: National Tuberculosis Reference Laboratory; Lab: Laboratory; CDC: Centers for Disease Control and Prevention; WHO: World Health Organization

\section{Declarations}

\section{Acknowledgements}

We thank Iganga Hospital staff from the laboratory for supporting the team during data collection. We acknowledge the Ministry of Health for authorization to collect data. We greatly appreciate technical guidance from CDC Atlanta team and Public Health Fellowship Program staff towards making the investigation a success.

\section{Funding}

This project was supported by the CDC Foundation through the Global TB Branch, Division of Global HIV and TB of the United States Centers for Disease Control and Prevention channeled via African Field Epidemiology Network 
and Makerere University School of Public Health to the Uganda Public Health Fellowship Program, Ministry of Health.

\section{Availability of data and materials}

The data from this investigation belong to the Uganda Public Health Fellowship Program and can be accessed upon reasonable request from the corresponding author and with permission from the Uganda Public Health Fellowship Program.

\section{Author contributions}

AM, LB, JM, and ARA, conceived and designed the study. AM and LB participated in data collection, cleaning, and analysis. AM prepared the original manuscript. LB, ARA, PC, and LS participated in manuscript writing and review to ensure scientific integrity and intellectual content. All authors read and approved the final manuscript.

\section{Ethical clearance and consent to participate}

The MoH Uganda through the office of the Director General of Health Services gave the directive and approval to conduct the study. Additionally, the office of the Associate Director for Science, US Centers for Disease Control and Prevention, Center for Global Health, Atlanta GA USA, determined that this investigation was not human subjects' research and issued Institutional review approval No: CGH-FETPT-7/23/19-dd2f9 for project ID $0900 f 3 e b 819 d d 2 f 9$. We used the patients' TB register numbers as the unique patient identifiers so that laboratory data (such as microscopy result, culture results, DST results, and all subsequent test results) and patient data forms could be matched; no data collection forms had patient names on them. All electronic data was de-identified and password protected on a password protected computer. We ensured confidentiality of data by keeping it under lock and key to avoid disclosure of personal information of the respondents to members who were not part of the investigation.

\section{Consent for publication}

Not applicable.

\section{Competing interests}

Authors declare no competing interests.

\section{Disclaimer}

The findings and conclusions in this report are those of the authors and do not necessarily represent the official position of the US Centers for Disease Control and Prevention, African Field Epidemiology Network, Makerere University School of Public Health, or Uganda Ministry of Health. 


\section{References}

1. WHO. WHO Global tuberculosis report 2017 [Internet]. World Health Organization Press. 2017. Available from: http://www.who.int/tb/publications/global_report/en/

2. WHO. Global Tuberculosis Report 2014. WHO Rep [Internet]. 2014;2014(4):171.

3. Zumla A, Abubakar I, Raviglione M, Hoelscher M, Ditiu L, McHugh TD, et al. Drug-Resistant tuberculosis-current dilemmas, unanswered questions, challenges, and priority needs. J Infect Dis. 2012;205(SUPPL. 2).

4. Rajendra Prasad, Nikhil Gupta 1 and Amitabh Banka. Multidrug-resistant tuberculosis/rifampicin-resistant tuberculosis: Principles of management. Lung India. 2018;35(1):78-81.

5. Lange C, Abubakar I, Alffenaar JWC, Bothamley G, Caminero JA, Carvalho ACC, et al. Management of patients with multidrugresistant/ extensively drug-resistant tuberculosis in Europe: A TBNET consensus statement. Eur Respir J [Internet]. 2014;44(1):23-63. Available from:

http://erj.ersjournals.com/content/44/1/23.full.pdf+html http://ovidsp.ovid.com/ovidweb.cgi?

$\mathrm{T}=\mathrm{JS} \& \mathrm{CSC}=\mathrm{Y} \& N E W S=\mathrm{N} \& P A G E=$ fulltext\&D$=$ emed $16 \& A N=373467678$

6. Zetola NM, Shin SS, Tumedi KA, Moeti K, Ncube R, Nicol M, et al. Mixed Mycobacterium tuberculosis complex infections and false-negative results for rifampin resistance by genexpert MTB/RIF are associated with poor clinical outcomes. J Clin Microbiol. 2014;52(7):2422-9.

7. Kim YW, Seong MW, Kim TS, Yoo CG, Kim YW, Han SK, et al. Evaluation of Xpert® MTB/RIF assay: Diagnosis and treatment outcomes in rifampicin-resistant tuberculosis. Int J Tuberc Lung Dis. 2015;19(10):1216-21.

8. Boehme CC, Nabeta P, Hillemann D, Nicol MP, Shenai S, Krapp F, et al. Rapid Molecular Detection of Tuberculosis and Rifampin Resistance. N Engl J Med [Internet]. 2010;363(11):1005-15. Available from: http://www.nejm.org/doi/abs/10.1056/NEJMoa0907847

9. Weyer K, Mirzayev F, Migliori GB, Van Gemert W, D’Ambrosio L, Zignol M, et al. Rapid molecular TB diagnosis: Evidence, policy making and global implementation of Xpert MTB/RIF. Vol. 42, European Respiratory Journal. 2013. p. 252-71.

10. Mukherjee JS, Rich ML, Socci AR, Joseph JK, Alcántara Virú F, Shin SS, et al. Programmes and principles in treatment of multidrug-resistant tuberculosis. Lancet. 2004.

11. Wells CD, Cegielski JP, Nelson LJ, Laserson KF, Holtz TH, Finlay A, et al. HIV Infection and Multidrug-Resistant Tuberculosis-The Perfect Storm. J Infect Dis. 2007;

12. MOH. National TB and Leprosy Program guidelines. Kampala, Uganda. 2012.

13. Global laboratory initiative. GLI Practical Guide to TB Laboratory Strengthening [Internet]. 2017 [cited 2019 May 20]. Available from: http://www.stoptb.org/wg/gli/assets/documents/GLI_practical_guide.pdf

14. Nakwon Kwak, Sun Mi Choi, Jinwoo Lee, Young Sik Park, Chang-Hoon Lee, Sang-Min Lee, Chul-Gyu Yoo, Young Whan Kim, Sung Koo Han and J-JY. Diagnostic Accuracy and Turnaround Time of the Xpert MTB/RIF Assay in Routine Clinical Practice. PLoS One. 2013;8(10).

15. Hanrahan CF, Haguma P, Ochom E, Kinera I, Cobelens F, Cattamanchi A, et al. Implementation of Xpert MTB/RIF in Uganda: Missed opportunities to improve diagnosis of tuberculosis. Open Forum Infect Dis [Internet]. 2016;3(2). Available from: https://www.scopus.com/inward/record.uri?eid=2-s2.0-

84995699975\&doi=10.1093\%2Fofid\%2Fofw068\&partnerID=40\&md5=3c3d542a856d11aab70de900c3c33a59

16. Hossain ST, Isaakidis P, Sagili KD, Islam S, Islam MA, Shewade HD, et al. The multi-drug resistant tuberculosis diagnosis and treatment cascade in Bangladesh. PLoS One. 2015; 
17. Yagui M, Perales MT, Asencios L, Vergara L, Suarez C, Yale G, et al. Timely diagnosis of MDR-TB under program conditions: Is rapid drug susceptibility testing sufficient? Int J Tuberc Lung Dis. 2006;

\section{Figures}

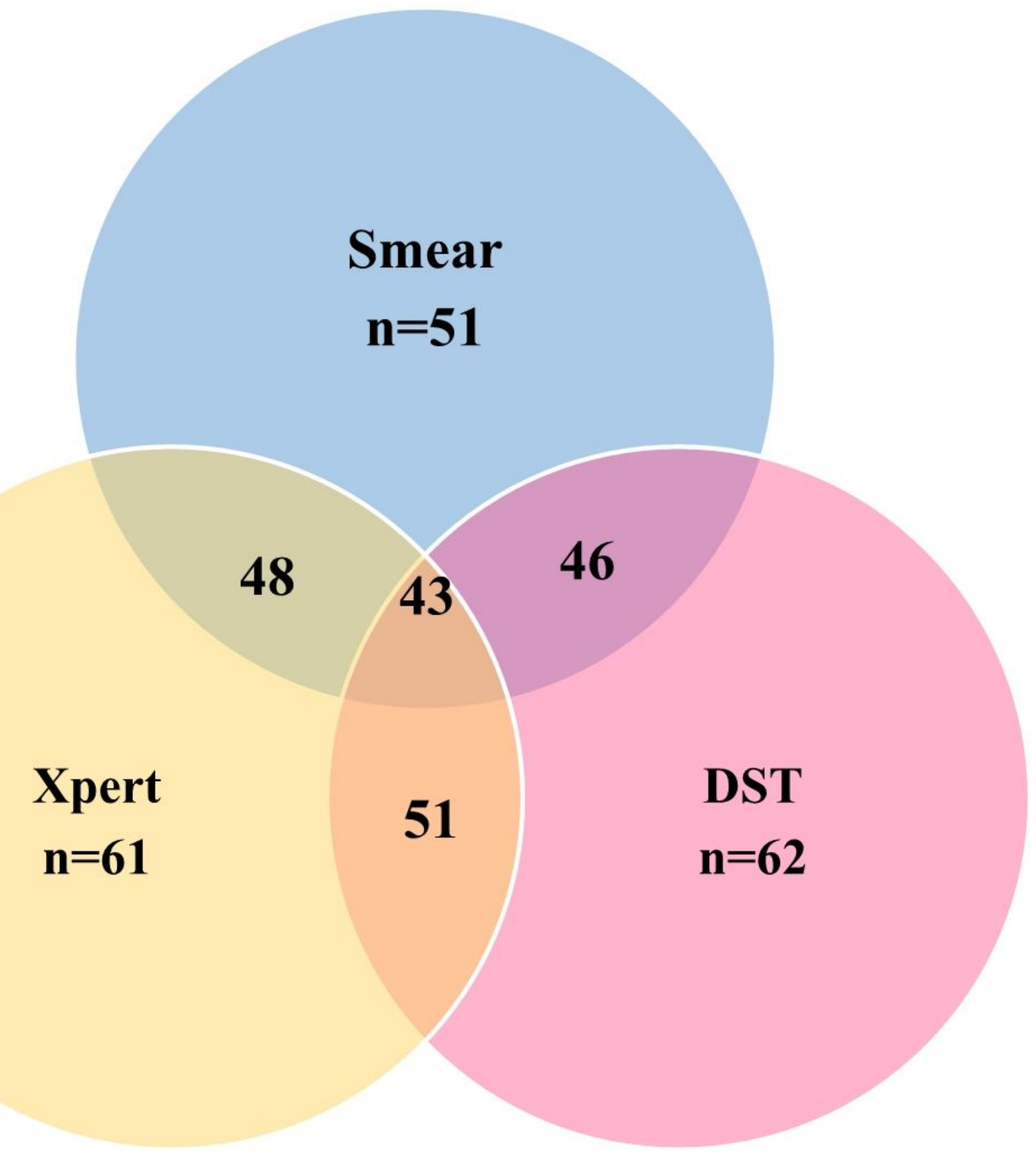

Figure 1

Number of eligible patient records reviewed having results for each of the three tests in Iganga Hospital from 20122017 
Median turnaround time

(days)

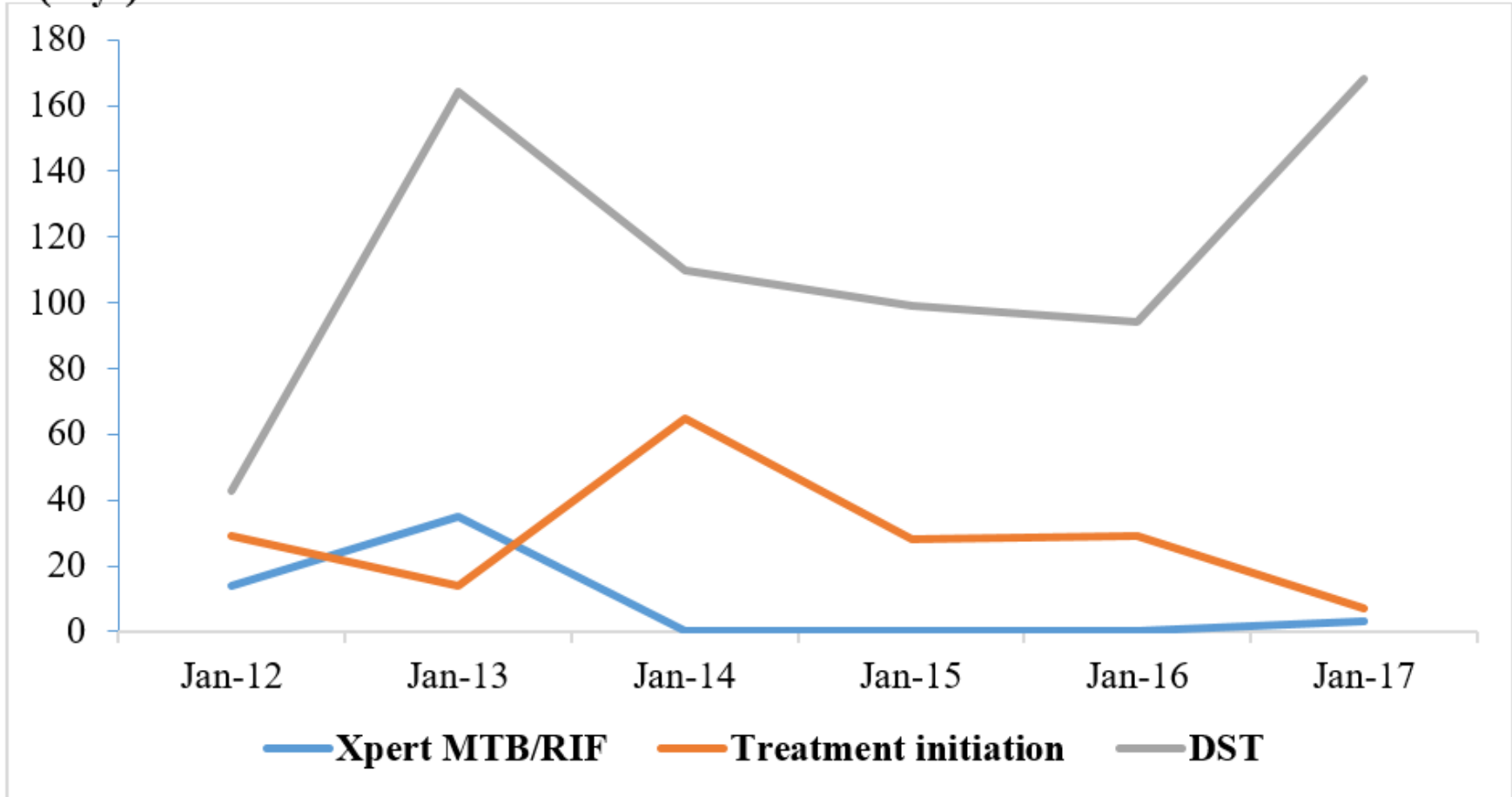

\section{Figure 2}

Trends of turnaround time for Xpert MTB/RIF, Drug Susceptibility Testing, and time to initiation on second line treatment, Iganga Hospital, multi-drug resistant tuberculosis treatment unit, 2012-2017. 


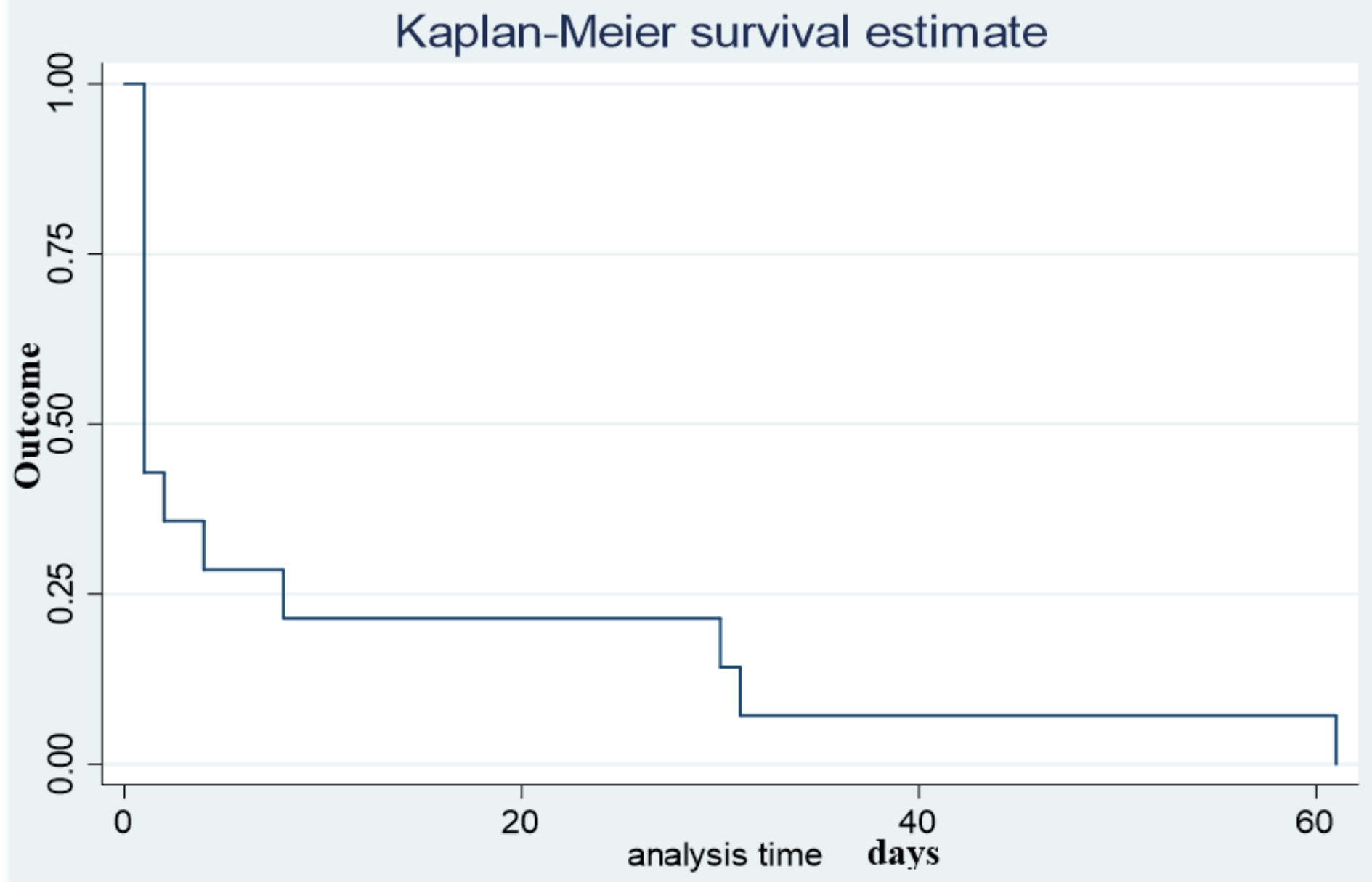

Figure 3

Kaplan Meier survival curve showing the turnaround time for smear microscopy from sample collection to reporting results for smear microscopy, Iganga Hospital multi-drug resistant tuberculosis treatment unit, 2012-2017 


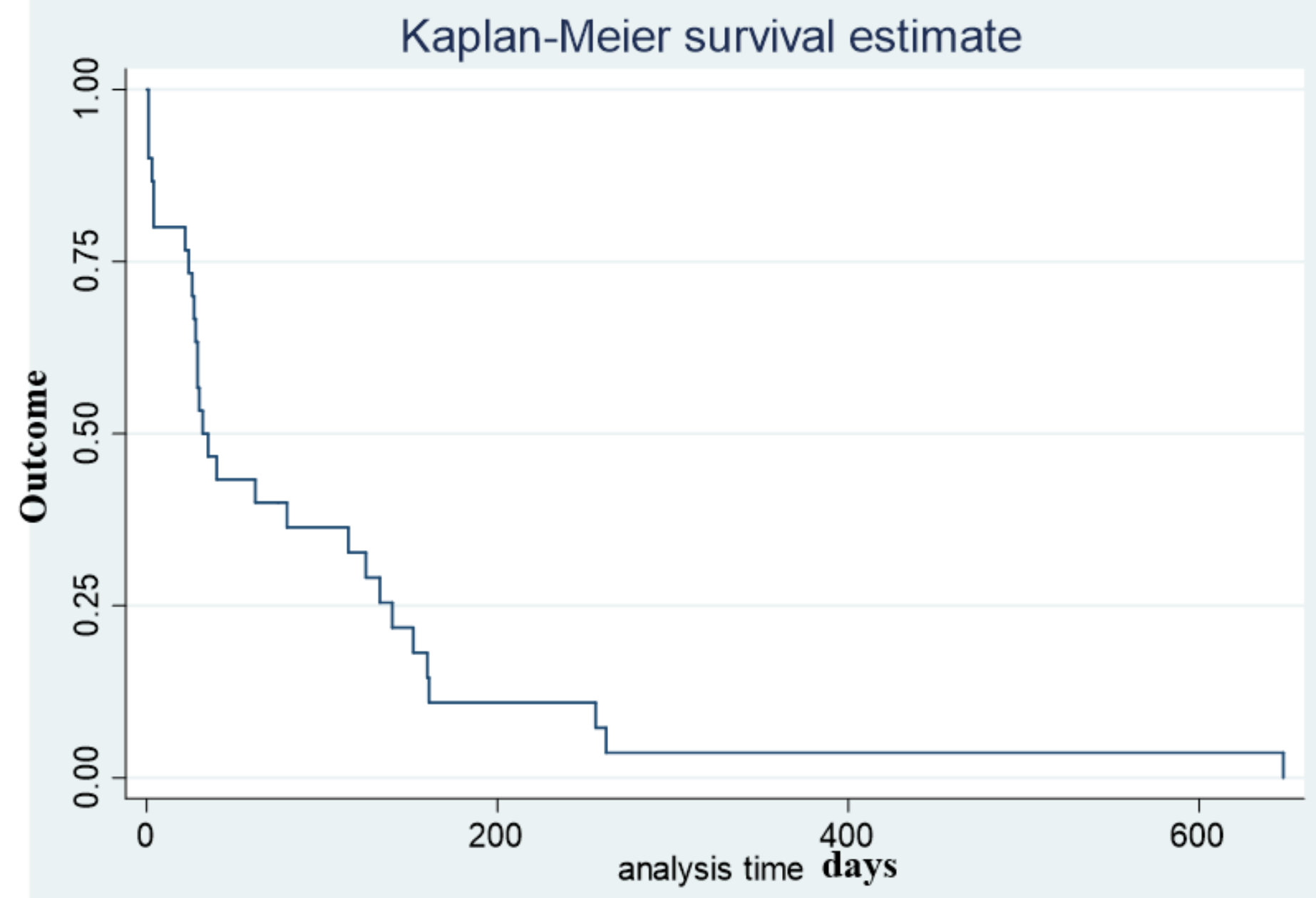

Figure 4

Kaplan Meier survival curve of turnaround time from when the sample was collected to when Xpert results are reported to referral facilities, 2012-2017 


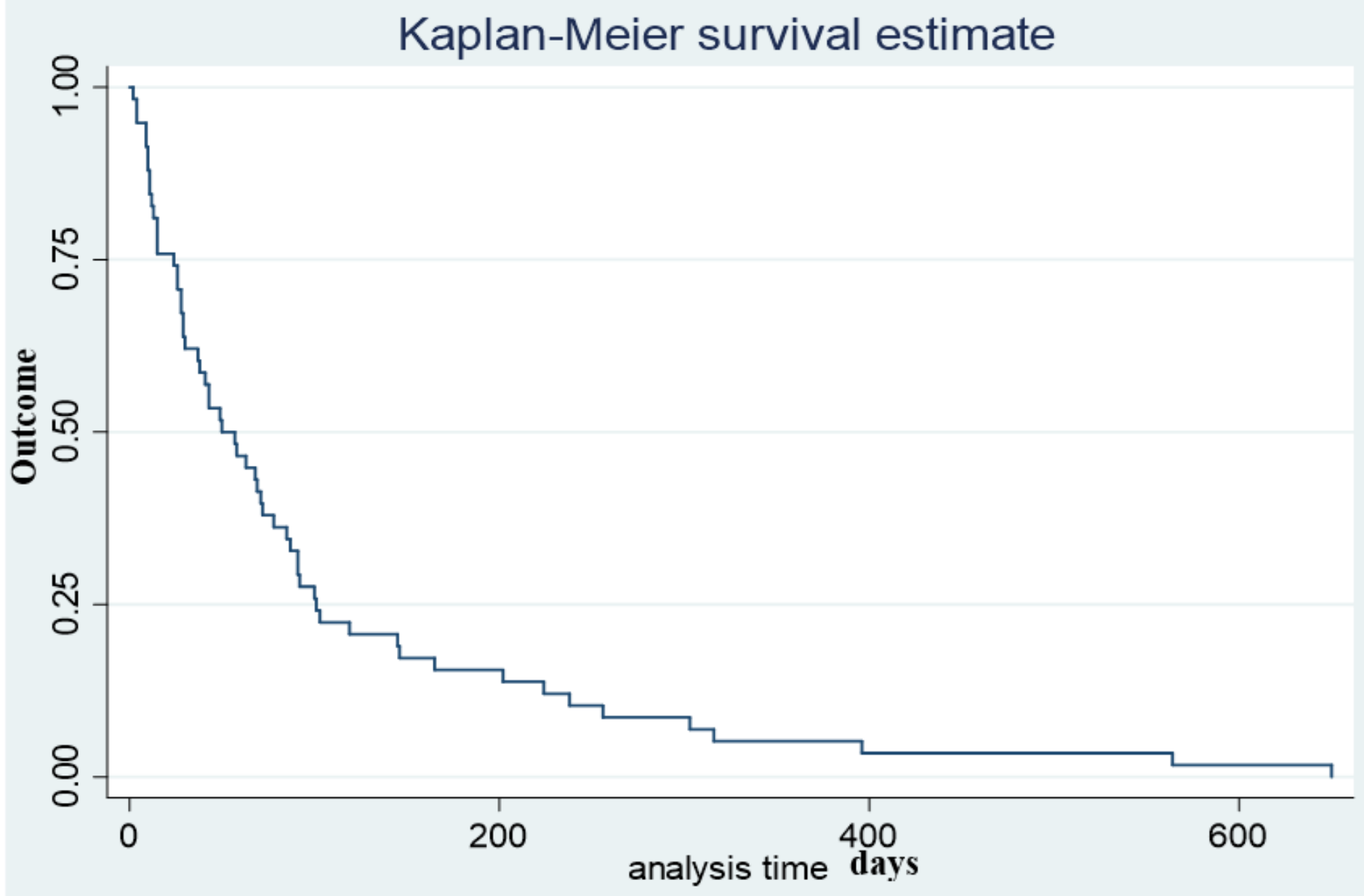

Figure 5

Kaplan Meier curves comparing survival analysis and turnaround time from when Xpert results are received to when patients are initiated on second line treatment, Iganga Hospital multi-drug resistant tuberculosis treatment unit, 2012-2017 